\title{
Single-Species Reactions on a Random Catalytic Chain.
}

\author{
G.Oshanin ${ }^{1}$ and S.F.Burlatsky² \\ ${ }^{1}$ Laboratoire de Physique Théorique des Liquides, \\ Université Paris 6, 4 Place Jussieu, 75252 Paris, France \\ ${ }^{2}$ United Technologies Research Center, \\ United Technologies Corporation, \\ 411 Silver Lane, 129-21 East Hartford, CT 06108, USA
}

\begin{abstract}
We present an exact solution for a catalytically-activated annihilation $A+A \rightarrow 0$ reaction taking place on a one-dimensional chain in which some segments (placed at random, with mean concentration $p$ ) possess special, catalytic properties. Annihilation reaction takes place, as soon as any two $A$ particles land from the reservoir onto two vacant sites at the extremities of the catalytic segment, or when any $A$ particle lands onto a vacant site on a catalytic segment while the site at the other extremity of this segment is already occupied by another $A$ particle. We find that the disorderaverage pressure $P^{(\text {quen })}$ per site of such a chain is given by $P^{(\text {quen })}=P^{(\text {lan })}+\beta^{-1} F$, where $P^{(\operatorname{lan})}=\beta^{-1} \ln (1+z)$ is the Langmuir adsorption pressure, $(z$ being the activity and $\beta^{-1}$ - the temperature), while $\beta^{-1} F$ is the reaction-induced contribution, which can be expressed, under appropriate change of notations, as the Lyapunov exponent for the product of $2 \times 2$ random matrices, obtained exactly by Derrida and Hilhorst (J. Phys. A 16, 2641 (1983)). Explicit asymptotic formulae for the particle mean density and the compressibility are also presented.
\end{abstract}

PACS numbers: 82.65.+r; 64.60.Cn; 68.43.De 


\section{Introduction.}

Catalytically-activated reactions (CARs), i.e. reactions between chemically inactive molecules which recombine only when some third substance - the catalytic substrate - is present, are widespread in nature $[1,2]$. Recently, such reactions have attracted a considerable attention following an early observation [3] of remarkable non-mean-field behavior exhibited by a specific reaction - the CO-oxidation in the presence of metal surfaces with catalytic properties $[1,2]$. An extensive analysis of this CAR has substantiated the emergence of an essentially different behavior as compared to the predictions of the classical, formal-kinetics scheme and have shown that under certain conditions such collective phenomena as phase transitions or the formation of bifurcation patterns may take place [3]. Prior to these works on catalytic systems, anomalous behavior was amply demonstrated in other schemes [4], involving reactions on contact between two particles at any point of the reaction volume (i.e., the "completely" catalytic sysems). It was realized [4] that the departure from the text-book, formal-kinetic predictions is due to many-particle effects, associated with fluctuations in the spatial distribution of the reacting species. This suggests that similarly to such "completely" catalytic reaction schemes, the behavior of the CARs may be influenced by many-particle effects.

Apart from the many-particle effects, behavior of the CARs in practically involved systems might be affected by the very structure of the catalytic substrate, which is often not well-defined geometrically, but must be viewed as an assembly of mobile or localized catalytic sites or islands, whose spatial distribution is complex [1]. Metallic catalysts, for instance, are often disordered compact aggregates, the building blocks of which are imperfect crystallites with broken faces, kinks and steps. Another example is furnished by porous materials with convoluted surfaces, such as, e.g., silica, alumina or carbons. Here the effective catalytic substrate is also only a portion of the total surface area because of the selective participation of different surface sites to the reaction. Finally, for liquid-phase CARs the catalyst can consist of active groups attached to polymer chains in solution.

Such complex morphologies render the theoretical analysis difficult. As yet, only em- 
pirical approaches have been used to account for the impact of the geometrical complexity on the behavior of the CARs, based mostly on heuristic concepts of effective reaction order or on phenomenological generalizations of the formal-kinetic "law of mass action" (see, e.g. Refs.[1] and [2] for more details). In this regard, analytical solutions of even somewhat idealized or simplified models, such as, for instance, the ones proposed in Refs.[3], are already highly desirable since such studies may provide an understanding of the effects of different factors on the properties of the CARs.

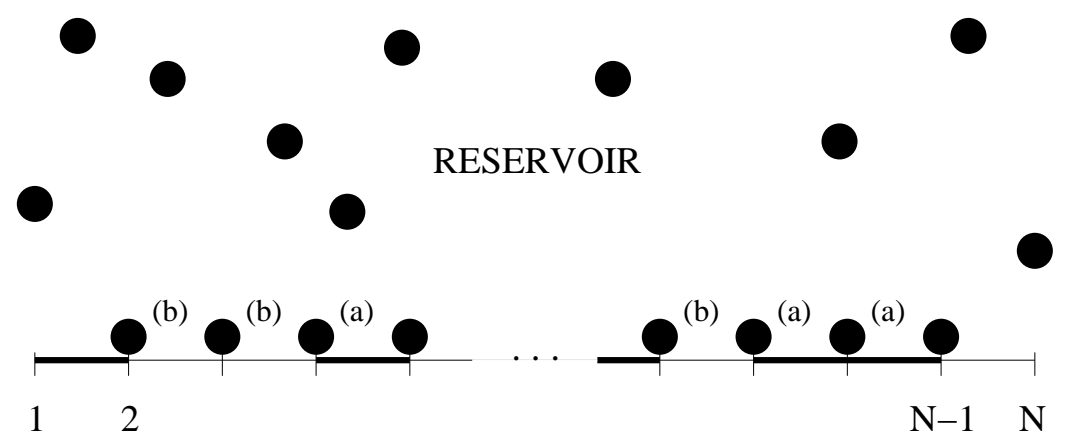

Figure 1: One-dimensional lattice of adsorption sites in contact with a reservoir. Filled circles denote hard-core $A$ particles. Thick black lines denote the segments with catalytic properties. (a) denotes a "forbidden" particle configuration, which corresponds to immediate reaction. (b) depicts the situation in which two neighboring $A$ particles may harmlessly coexist.

In this paper we study a catalytically-activated annihilation $A+A \rightarrow 0$ reaction in a simple, one-dimensional model with random distribution of the catalyst, appropriate to the just mentioned situation with the catalytically-activated reactions on polymer chains. We present here an exact solution for this model with quenched random distribution of the catalyst and show that despite its apparent simplicity it exhibits an interesting non-trivial behavior. We note finally that kinetics of $A+A \rightarrow 0$ reactions involving diffusive $A$ particles which react upon encounters on randomly placed catalytic sites has been discussed already in Refs. [5-7] and [8], and a rather surprising behavior has been found, especially in low-dimensional systems. Additionally, steady-state properties of $A+A \rightarrow 0$ reactions between immobile $A$ particles with long-range reaction probabilities in systems with external 
particles input have been presented in Refs. [9] and [10] and revealed non-trivial ordering phenomena with anomalous input intensity dependence of the mean particle density, which agrees with experimental observations [11].

\section{The model.}

Consider a one-dimensional regular lattice of unit spacing comprising $N$ adsorption sites. The lattice is in contact with a reservoir of identical, non-interacting hard-core $A$ particles (see, Fig.1) - a vapor phase, which is steadily maintained at a constant pressure.

The $A$ particles from the vapor phase can adsorb onto vacant adsorption sites and desorb back to the reservoir. The occupation of the " $i$ "-th adsorption site is described by the Boolean variable $n_{i}$, such that

$$
n_{i}=\left\{\begin{array}{l}
1, \text { if the "i"-th site is occupied } \\
0, \text { otherwise. }
\end{array}\right.
$$

Suppose next that some of the segments - intervals between neighboring adsorption sites possess "catalytic" properties (thick black lines in Fig.1) in the sense that they induce an immediate reaction $A+A \rightarrow 0$, as soon as two $A$ particles land onto two vacant sites at the extremities of the catalytic segment, or an $A$ particle lands onto a vacant site at one extremety of the catalytic segment while the site at the other extremity of this segment is already occupied by another $A$ particle. Two reacted $A$ particles instantaneously leave the lattice (desorb back to the reservoir). Any two $A$ particle adsorbed at extremities of a non-catalytic segment harmlessly coexist.

To specify the positions of the catalytic segments, we introduce a Boolean variable $\zeta_{i}$, so that $\zeta_{0}=\zeta_{N}=0$ and

$$
\zeta_{i}=\left\{\begin{array}{l}
1, \text { if the } i \text {-t interval is catalytic, } i=1,2, \ldots, N-1, \\
0, \text { otherwise. }
\end{array}\right.
$$

In what follows we suppose that $\zeta_{i}$ are independent, identically distributed quenched ran- 
dom variables with distribution

$$
\rho(\zeta)=p \delta(\zeta-1)+(1-p) \delta(\zeta)
$$

Now, for a given distribution of the catalytic segments, the partition function $Z_{N}(\zeta)$ of the system under study can be written as follows:

$$
Z_{N}(\zeta)=\sum_{\left\{n_{i}\right\}} z^{\sum_{i=1}^{N} n_{i}} \prod_{i=1}^{N-1}\left(1-\zeta_{i} n_{i} n_{i+1}\right),
$$

where the summation $\sum_{\left\{n_{i}\right\}}$ extends over all possible configurations $\left\{n_{i}\right\}$, while

$$
z=\exp (\beta \mu)
$$

is the activity and $\mu$ - the chemical potential. Note that $Z_{N}(\zeta)$ in Eq.(2) is a functional of the configuration $\zeta=\left\{\zeta_{i}\right\}$.

It is worth-while to remark that $Z_{N}(\zeta)$ can also be thought of as a one-dimensional version of models describing adsorption of hard-molecules [12-19], i.e. adsorption limited by the "kinetic" constraint that any two molecules can neither occupy the same site nor appear on the neighboring sites. The most celebrated examples of such models are furnished by the so-called "hard-squares" model [12-16], or by the "hard-hexagons" model first solved exactly by Baxter [18]. In our case of the CARs on random catalytic substrates the nearest-neighbor exclusion constraint is introduced only locally, at some specified, randomly distributed intervals. Such locally frustrated models of random reaction/adsorption thus represent a natural and meaningful generalization of the well-studied exclusion models over systems with disorder. Of course, in this context two-dimensional situations are of most interest, but nonetheless it might be instructive to find examples of such models which can be solved exactly in one dimension.

Our main goal here is to calculate the disorder-average pressure per site:

$$
P^{(\text {quen })}=\frac{1}{\beta} \lim _{N \rightarrow \infty} \frac{1}{N}\left\langle\ln \left(Z_{N}(\zeta)\right)\right\rangle_{\zeta}
$$

where the angle brackets with the subscript $\zeta$ denote averaging over all possible configurations $\left\{\zeta_{i}\right\}$. Once $P^{(q u e n)}$ is obtained, all other pertinent thermodynamic properties can 
be readily evaluated by differentiating $P^{(q u e n)}$ with respect to the chemical potential $\mu$; in particular, the disorder-average mean particle density $n^{(q u e n)}$ will be given by

$$
n^{(q u e n)}=\frac{\partial}{\partial \mu} P^{(q u e n)}
$$

while the compressibility $k_{T}$ obeys

$$
k_{T}^{(\text {quen })}=\frac{1}{\left(n^{(q u e n)}\right)^{2}} \frac{\partial n^{(q u e n)}}{\partial \mu} .
$$

To close this section, we display the results corresponding to two "regular" cases: namely, when $p=0$ and $p=1$, which will serve us in what follows as some benchmarks. In the $p=0$ all sites are decoupled, and one has the Langmuir results:

$$
P^{(\text {lan })}=\frac{1}{\beta} \ln (1+z), \quad n^{(\text {lan })}=\frac{z}{1+z}, \quad \text { and } \quad \beta^{-1} k_{T}^{(\text {lan })}=\frac{1}{z}
$$

The "regular" case when $p=1$ is a bit less trivial, but the solution can be still straightforwardly obtained. In this case, we have

$$
P^{(r e g)}=\frac{1}{\beta} \ln \left(\frac{\sqrt{1+4 z}+1}{2}\right), \quad n^{(r e g)}=1-\frac{2 z}{1+4 z-\sqrt{1+4 z}},
$$

and

$$
\beta^{-1} k_{T}^{(r e g)}=\frac{2 z}{\sqrt{1+4 z}(1+2 z-\sqrt{1+4 z})} .
$$

Note that in the $p=1$ case (the completely catalytic system) the mean particle density

tends to $1 / 2$ as $z \rightarrow \infty$ (compared to $n^{(\operatorname{lan})} \rightarrow 1$ behavior observed for the Langmuir case), which means that the adsorbent undergoes "ordering" transition and particles distribution on the lattice becomes periodic revealing a spontaneous symmetry breaking between two sublattices. In the limit $z \rightarrow \infty$ the compressibility vanishes as $k_{T}^{(\text {reg })} \propto 1 / \sqrt{z}$ compared to the Langmuir behavior $k_{T}^{(\text {lan })} \propto 1 / z$.

\section{Recursion relations for $Z_{N}(\zeta)$.}

Let us first introduce an auxiliary, constrained partition function of the form

$$
Z_{N}^{\prime}(\zeta)=\left.Z_{N}(\zeta)\right|_{n_{N}=1}=z \sum_{\left\{n_{i}\right\}} z^{\sum_{i=1}^{N-1} n_{i}} \prod_{i=1}^{N-2}\left(1-\zeta_{i} n_{i} n_{i+1}\right)\left(1-\zeta_{N-1} n_{N-1}\right)
$$


i.e. $Z_{N}^{\prime}(\zeta)$ stands for the partition function of a system with fixed set $\zeta=\left\{\zeta_{i}\right\}$ and fixed occupation of the site $i=N, n_{N}=1$. Evidently, we have that

$$
Z_{N}(\zeta)=Z_{N-1}(\zeta)+Z_{N}^{\prime}(\zeta)
$$

Next, considering two possible values of the occupation variable $n_{N-1}$, i.e. $n_{N-1}=0$ and $n_{N-1}=1$, we find that $Z_{N}^{\prime}(\zeta)$ can be expressed through $Z_{N-2}(\zeta)$ and $Z_{N-1}^{\prime}(\zeta)$ as

$$
\begin{aligned}
Z_{N}^{\prime}(\zeta) & =z \sum_{\left\{n_{i}\right\}} z^{\sum_{i=1}^{N-2} n_{i}} \prod_{i=1}^{N-3}\left(1-\zeta_{i} n_{i} n_{i+1}\right)+ \\
& +z^{2}\left(1-\zeta_{N-1}\right) \sum_{\left\{n_{i}\right\}} z^{\sum_{i=1}^{N-2} n_{i}} \prod_{i=1}^{N-3}\left(1-\zeta_{i} n_{i} n_{i+1}\right)\left(1-\zeta_{N-2} n_{N-2}\right)= \\
& =z Z_{N-2}(\zeta)+z\left(1-\zeta_{N-1}\right) Z_{N-1}^{\prime}(\zeta)
\end{aligned}
$$

Now, recursion in Eq.(11) allows us to eliminate $Z_{N}^{\prime}(\zeta)$ in Eq.(12). From Eq.(11) we have $Z_{N}^{\prime}(\zeta)=Z_{N}(\zeta)-Z_{N-1}(\zeta)$, and consequently, we find from Eq.(12) that the unconstrained partition function $Z_{N}(\zeta)$ in Eq.(2) obeys the following recursion

$$
Z_{N}(\zeta)=\left(1+z\left(1-\zeta_{N-1}\right)\right) Z_{N-1}(\zeta)+z \zeta_{N-1} Z_{N-2}(\zeta)
$$

which is to be solved subject to evident initial conditions

$$
Z_{0}(\zeta) \equiv 1 \text { and } \quad Z_{1}(\zeta) \equiv 1+z
$$

A conventional way (see, e.g. Ref.[20-22]) to study linear random three-term recursions is to reduce them to random maps by introducing the Ricatti variable of the form

$$
R_{N}(\zeta)=\frac{Z_{N}(\zeta)}{Z_{N-1}(\zeta)}
$$

In terms of this variable Eq.(13) becomes

$$
R_{N}(\zeta)=\left(1+z\left(1-\zeta_{N-1}\right)\right)+\frac{z \zeta_{N-1}}{R_{N-1}(\zeta)}, \quad \text { with } \quad R_{1}(\zeta) \equiv R_{1}=1+z
$$

which represents a random homographic relation. Once $R_{N}(\zeta)$ is defined for arbitrary $N$, the partition function $Z_{N}(\zeta)$ can be readily determined as the product,

$$
Z_{N}(\zeta)=\prod_{i=1}^{N} R_{i}(\zeta)
$$


and hence, the disorder-average logarithm of the partition function will be obtained as

$$
\left\langle\ln Z_{N}(\zeta)\right\rangle_{\zeta}=\sum_{i=1}^{N}\left\langle\ln R_{i}(\zeta)\right\rangle_{\zeta}
$$

Before we proceed further on, we note that recursion schemes of quite a similar form have been discussed already in the literature in different contexts. In particular, two decades ago Derrida and Hilhorst [20] (see also Ref.[23] for a more general discussion) have shown that such recursions occur in the analysis of the Lyapunov exponent $F(\epsilon)$ of the product of random $2 \times 2$ matrices of the form

$$
F(\epsilon)=\lim _{N \rightarrow \infty} \frac{1}{N}\left\langle\ln \left(\operatorname{Tr}\left[\prod_{i=1}^{N}\left(\begin{array}{cc}
1 & \epsilon \\
z_{i} \epsilon & z_{i}
\end{array}\right)\right]\right)\right\rangle_{\left\{z_{i}\right\}},
$$

where $z_{i}$ are independent positive random numbers with a given probability distribution $\rho(z)$. Equation (19) is related, for instance, to the disorder-average free energy of an Ising chain with nearest-neighbor interactions in a random magnetic field, and appears in the solution of a two-dimensional Ising model with row-wise random vertical interactions [24], the role of $\epsilon$ being played by the wavenumber $\theta$. The recurence scheme in Eq.(16) emerges also in such an interesting context as the problem of enumeration of primitive words with random errors in the locally free and braid groups [25]. Some other examples of physical systems in which the recursion in Eq.(16) appears can be found in Ref.[22].

Further on, Derrida and Hilhorst [20] have demonstrated that $F(\epsilon)$ can be expressed as

$$
F(\epsilon)=\lim _{N \rightarrow \infty} \frac{1}{N} \sum_{i=1}^{N}\left\langle\ln R_{i}^{\prime}\right\rangle_{\left\{z_{i}\right\}},
$$

where $R_{i}^{\prime}$ are defined through the recursion

$$
R_{i}^{\prime}=1+z_{i-1}+z_{i-1}\left(\epsilon^{2}-1\right) / R_{i-1}^{\prime}, \quad \text { with } \quad R_{1}^{\prime}=1 .
$$

Moreover, they have shown that the model admits an exact solution when

$$
\rho(z)=(1-p) \delta(z)+p \delta(z-y),
$$


i.e. when similarly to the model under study, $z_{i}$ are independent, random two-state variables assuming only two values - $y$ with probability $p$ and 0 with probability $1-p$. Supposing that when $i$ increases, a stationary probability distribution $P\left(R^{\prime}\right)$ of the $R_{i}^{\prime}$ independent of $i$ exists [26], Derrida and Hilhorst [20] have found the following exact result:

$$
\begin{aligned}
F(\epsilon) & =p \ln (1+b)-p(2-p) \ln \left(1+b \frac{y-b}{1-b y}\right)+ \\
& +(1-p)^{2} \sum_{N=1}^{\infty} p^{N} \ln \left(1+b\left(\frac{y-b}{1-b y}\right)^{N+1}\right)
\end{aligned}
$$

where

$$
b=1+\frac{(1-y)^{2}}{2 \epsilon^{2} y}\left[1-\left(1+4 \frac{\epsilon^{2} y}{(1-y)^{2}}\right)^{1 / 2}\right] .
$$

\section{Disorder-average pressure.}

We turn now back to our recursion scheme in Eq.(16) and notice that setting

$$
R_{i}(\zeta)=(1+z) R_{i}^{\prime}
$$

and choosing

$$
y=-\frac{z}{1+z}=-n^{(l a n)}, \quad \text { and } \quad \epsilon^{2}=\frac{z}{1+z}=n^{(l a n)},
$$

makes the recursion schemes in Eqs.(16) and (21) identic! Consequently, the disorderaverage pressure per site in our random catalytically-activated reaction/adsorption model can be expressed as

$$
P^{(q u e n)} \equiv \frac{1}{\beta} \ln (1+z)+\frac{1}{\beta} F(\epsilon)
$$

where $F(\epsilon)$ is the Lyapunov exponent of the product of random $2 \times 2$ matrices in Eq.(19), in which $\epsilon$ and $z_{i}$ are defined by Eqs.(22) and (26).

Note next that the first term on the right-hand-side of Eq.(27) is a trivial Langmuir result for the $p=0$ case (adsorption without reaction) which would entail $n^{(q u e n)}=z /(1+$ $z)$, Eq.(7). Hence, all non-trivial, disorder-induced behavior is embodied in the Lyapunov exponent $F(\epsilon)$. 
The disorder-averaged pressure per site for the random reaction/adsorption model under study can be thus readily obtained from Eqs.(23) and (24) by defining the parameters $y$ and $\epsilon$ as prescribed in Eq.(26). This yields the following exact result:

$$
\begin{aligned}
\beta P^{(q u e n)} & =\ln \left(\phi_{z}\right)-(1-p) \ln \left(1-\omega^{2}\right)+ \\
& +\frac{(1-p)^{2}}{p} \sum_{N=1}^{\infty} p^{N} \ln \left(1-(-1)^{N} \omega^{N+2}\right),
\end{aligned}
$$

where

$$
\phi_{z}=\frac{1+\sqrt{1+4 z}}{2}
$$

and

$$
\omega=\frac{\sqrt{1+4 z}-1}{\sqrt{1+4 z}+1}=z / \phi_{z}^{2}=1-\frac{1}{\phi_{z}}
$$

Note that $\phi_{z}$ obeys $\phi_{z}\left(\phi_{z}-1\right)=z$; hence, $\phi_{z=1}=(\sqrt{5}+1) / 2$ is just the "golden mean".

\section{Asymptotic behavior of the disorder-average pres- sure, mean density and the compressibility.}

Consider first the asymptotic behavior of $P^{(q u e n)}$ in the small- $z$ limit. To do this, it is expedient to use another representation of $P^{(q u e n)}$. After some straightforward calculations, one can cast $P^{(q u e n)}$ in Eq.(28) into the form:

$$
\beta P^{(q u e n)}=\frac{(1-p)}{p} \sum_{n=0}^{\infty} p^{n} \mathcal{F}_{n},
$$

where $\mathcal{F}_{n}$ denote natural logarithms of the Stieltjes-type continued fractions of the form

$$
\mathcal{F}_{n}=\ln \left(1+\frac{z}{1+\frac{z}{1+\frac{z}{1+\frac{\cdots}{1+z}}}}\right) .
$$

Note now that in the limit $n \rightarrow \infty$, one has

$$
\lim _{n \rightarrow \infty} \mathcal{F}_{n}=\ln \left(\phi_{z}\right)=\ln \left(\frac{1+\sqrt{1+4 z}}{2}\right)
$$


i.e. $\mathcal{F}_{n}$ is the $n$-th approximant of $\ln \left(\phi_{z}\right)$; hence, $P^{(q u e n)}$ can be thought of as the generating function of such approximants. Now, one finds that for $z<1$ the sequence of approximants converges quickly to $\ln \left(\phi_{z}\right)$; expanding the $n$-th approximant $\mathcal{F}_{n}$ into the Taylor series in powers of $z$, one has that the first $n$ terms of such an expansion coincide with the first $n$ terms of the expansion of $\ln \left(\phi_{z}\right)$, i.e.

$$
\ln \left(\phi_{z}\right)=\ln \left(\frac{1+\sqrt{1+4 z}}{2}\right)=-\frac{1}{2 \sqrt{\pi}} \sum_{n=1}^{\infty} \frac{(-1)^{n} \Gamma(n+1 / 2)}{\Gamma(n+1)} \frac{(4 z)^{n}}{n},
$$

Consequently, $\mathcal{F}_{n}$ and $\mathcal{F}_{n-1}$ differ only by terms of order $z^{n}$, which signifies that convergence is good. On the other hand, for $z \geq 1$ convergence becomes poor and one has to seek for a more suitable representation. As a matter of fact, already for $z=1$ one has that in the limit $n \rightarrow \infty$ the approximant $\mathcal{F}_{n}$ tends to $\ln \left(\phi_{1}\right)$, i.e. the logarithm of the "golden mean", which is known as the irrational number worst approximated by rationals.

In the small- $z$ limit, we find then using an expansion in Eq.(34) that $P^{(q u e n)}$ follows

$$
\beta P^{(\text {quen })}=z-\left(\frac{1}{2}+p\right) z^{2}+\left(\frac{1}{3}+2 p+p^{2}\right) z^{3}-\left(\frac{1}{4}+\frac{7}{2} p+4 p^{2}+p^{3}\right) z^{4}+\mathcal{O}\left(z^{5}\right) .
$$

Consequently, in the small- $z$ limit the mean density obeys:

$$
n^{(q u e n)}=z-(1+2 p) z^{2}+\left(1+6 p+3 p^{2}\right) z^{3}-\left(1+14 p+16 p^{2}+4 p^{2}\right) z^{4}+\mathcal{O}\left(z^{5}\right),
$$

while the compressibility $k_{T}^{(q u e n)}$ is given by

$$
\beta^{-1} k_{T}^{(\text {quen })}=\frac{1}{z}+p(2-p) z-4 p(2-p) z^{2}+3 p\left(8-p-2 p^{2}\right) z^{3}+\mathcal{O}\left(z^{4}\right) .
$$

Note that, the coefficients in the small- $z$ expansion coincide with the coefficients in the expansions of $P^{(l a n)}$ and $P^{(r e g)}$ when we set in Eq.(35) $p=0$ or $p=1$.

Now, we turn to the analysis of the large- $z$ behavior which is a bit more complex than $z \ll 1$ case and requires understanding of the asymptotic behavior of the sum

$$
S=\sum_{N=1}^{\infty} p^{N} \ln \left(1-(-1)^{N} \omega^{N+2}\right)
$$

entering Eq.(28). We note first that in this sum the behavior of the terms with odd and even $N$ is quite different and we have to consider it separately. Let

$$
S_{\text {odd }}=\frac{1}{p} \sum_{N=1}^{\infty} p^{2 N} \ln \left(1+\omega^{2 N+1}\right)
$$


denote the contribution of the terms with odd $N$. Note that when $z \rightarrow \infty$ (i.e. $\omega \rightarrow 1$ ) the sum $S_{\text {odd }}$ tends to $p \ln (2) /\left(1-p^{2}\right)$. The corrections to this limiting behavior can be defined as follows. Expanding $\ln \left(1+\omega^{2 N+1}\right)$ into the Taylor series in powers of $\omega$ and then, using the definition $\omega=1-1 / \phi_{z}$ and the binomial expansion, we construct a series in the inverse powers of $\phi_{z}$ :

$$
\begin{aligned}
S_{\text {odd }} & =\frac{p}{1-p^{2}} \ln (2)-\frac{1}{2} \frac{p\left(3-p^{2}\right)}{\left(1-p^{2}\right)^{2}} \frac{1}{\phi_{z}}+ \\
& +\frac{1}{8} \frac{p\left(3+6 p^{2}-p^{4}\right)}{\left(1-p^{2}\right)^{3}} \frac{1}{\phi_{z}^{2}}+\frac{1}{24} \frac{p\left(15+10 p^{2}-p^{4}\right)}{\left(1-p^{2}\right)^{3}} \frac{1}{\phi_{z}^{3}}+\mathcal{O}\left(\frac{1}{\phi_{z}^{4}}\right)
\end{aligned}
$$

Note that this expansion is only meaningful when $\phi_{z} \gg(1-p)^{-1},\left(z \gg(1-p)^{-2}\right)$, which signifies that $p=1$ is a special point.

Further on, plugging into the latter expansion the definition of $\phi_{z}, \phi_{z}=(1+\sqrt{1+4 z}) / 2$, we obtain the following expansion in the inverse powers of the activity $z$ :

$$
\begin{aligned}
S_{\text {odd }} & =\frac{p}{1-p^{2}} \ln (2)-\frac{p}{2} \frac{\left(3-p^{2}\right)}{\left(1-p^{2}\right)^{2}} \frac{1}{z^{1 / 2}}+ \\
& +\frac{p}{8} \frac{\left(9-2 p^{2}+p^{4}\right)}{\left(1-p^{2}\right)^{3}} \frac{1}{z}+\frac{p}{48} \frac{\left(3-4 p^{2}+p^{4}\right)}{\left(1-p^{2}\right)^{3}} \frac{1}{z^{3 / 2}}+\mathcal{O}\left(\frac{1}{z^{2}}\right)
\end{aligned}
$$

Consider next the sum

$$
S_{\text {even }}=\sum_{N=1}^{\infty} p^{2 N} \ln \left(1-\omega^{2 N+2}\right),
$$

which represents the contribution of terms with even $N$. Note that in contrast to the behavior of $S_{\text {odd }}$, the sum in Eq. (42) diverges when $z \rightarrow \infty(\omega \rightarrow 1)$. Since $1-\omega^{2 N+2} \sim 1-\omega$ when $\omega \rightarrow 1$, we have that in this limit the leadin behavior of $S_{\text {even }}$ is described by

$$
S_{\text {even }} \sim \frac{p^{2}}{1-p^{2}} \ln (1-\omega) .
$$

To obtain several correction terms we make use of one of Gessel's expansions [27]:

$$
\ln \left(\frac{2(N+1) x}{1-(1-x)^{2 N+2}}\right)=\sum_{k=1}^{\infty} g_{k}(2 N+2) \frac{(-1)^{k} x^{k}}{k},
$$

where $g_{k}(2 N+2)$ are the Dedekind-type sums of the form

$$
g_{k}(2 N+2)=\sum_{\zeta^{2 N+2}=1, \zeta \neq 1} \frac{1}{(\zeta-1)^{k}},
$$


where the summation extends over all $\zeta$ being the $(2 N+2)$-th roots of unity (with $\zeta=1$ excluded). As shown in Ref.[27], the weights $g_{k}(2 N+2)$ are polynomials in $N$ of degree at most $k$ with rational coefficients. Next, setting $x=1 / \phi_{z}$ in the expansion in Eq.(44), plugging it to Eq.(42) and performing summations over $N$, we find that $S_{\text {even }}$ can be written down as

$$
S_{\text {even }}=-\frac{p^{2}}{1-p^{2}} \ln \left(\phi_{z}\right)+\frac{p^{2}}{1-p^{2}} \ln (2)+s_{p}-\sum_{k=1}^{\infty} G_{k}(p) \frac{(-1)^{k}}{k \phi_{z}^{k}}
$$

where $s_{p}$ is an infinite series of the form ${ }^{1}$

$$
s_{p}=\sum_{N=1}^{\infty} p^{2 N} \ln (N+1)
$$

while $G_{k}(p)$ are the generating functions of the polynomials $g_{k}(2 N+2)$ :

$$
G_{k}(p)=\sum_{N=1}^{\infty} g_{k}(2 N+2) p^{2 N}
$$

Inserting next the definition of $\phi_{z}$, we find the following explicit asymptotic expansion

$$
\begin{aligned}
S_{\text {even }} & =-\frac{1}{2} \frac{p^{2}}{1-p^{2}} \ln (z)+\frac{p^{2}}{1-p^{2}} \ln (2)+s_{p}+ \\
& -\frac{p^{2}\left(2-p^{2}\right)}{\left(1-p^{2}\right)^{2}} \frac{1}{z^{1 / 2}}+\frac{p^{2}\left(21-18 p^{2}+5 p^{4}\right)}{24\left(1-p^{2}\right)^{3}} \frac{1}{z}+\frac{p^{2}\left(2-p^{2}\right)}{24\left(1-p^{2}\right)^{2}} \frac{1}{z^{3 / 2}}+\mathcal{O}\left(\frac{1}{z^{2}}\right)
\end{aligned}
$$

Finally, combining the expansions in Eqs.(28), (41) and (50), we find the following large- $z$ expansion for the disorder-averaged pressure $P^{(q u e n)}$ :

$$
\begin{aligned}
\beta P^{(\text {quen })} & =\frac{1}{1+p} \ln (z)-\frac{(1-p)^{2}}{(1+p)} \ln (2)+ \\
& +\frac{(1-p)^{2}}{p} s_{p}+\frac{1}{6} \frac{6+3 p-p^{3}}{(1+p)^{2}\left(1-p^{2}\right)} \frac{1}{z}+\mathcal{O}\left(\frac{1}{z^{2}}\right)
\end{aligned}
$$

which yields

$$
n^{(\text {quen })}=\frac{1}{1+p}-\frac{1}{6} \frac{6+3 p-p^{3}}{(1+p)^{2}\left(1-p^{2}\right)} \frac{1}{z}+\mathcal{O}\left(\frac{1}{z^{2}}\right),
$$

${ }^{1}$ Note that $s_{p}$ shows a non-analytic behavior when $p \rightarrow 1$. This function can be represented as

$$
s_{p}=-\frac{1}{1-p^{2}} \ln \left(1-p^{2}\right)-\frac{p^{2}}{1-p^{2}} \sum_{n=2}^{\infty} \frac{(-1)^{n}}{n} \Phi\left(p^{2}, n, 1\right),
$$

where $\Phi\left(p^{2}, n, 1\right)$ are the Lerch transcedents, $\Phi\left(p^{2}, n, 1\right)=\sum_{l=0}^{\infty}(1+l)^{-n} p^{2 l}$. It is straightforward to find then that $s_{p}=-\frac{1}{1-p^{2}} \ln \left(1-p^{2}\right)-\frac{\gamma}{1-p^{2}}+\mathcal{O}(\ln (p))$, where $\gamma$ is the Euler constant. 
and

$$
\beta^{-1} k_{T}^{(q u e n)}=\frac{1}{6} \frac{6+3 p-p^{3}}{(1+p)\left(1-p^{2}\right)} \frac{1}{z}+\frac{1}{36} \frac{p\left(6+3 p-p^{3}\right)^{2}}{(1+p)^{2}\left(1-p^{2}\right)^{2}} \frac{1}{z^{2}}+\mathcal{O}\left(\frac{1}{z^{3}}\right) .
$$

Note that asymptotic expansions in Eqs.(51), (52) and (53) are only meaningful for $z \gg$ $(1-p)^{-2}$ and thus exclude the completely catalytic $p=1$ case. At this special point $p \equiv 1$ we find from Eqs.(8) that the pressure and mean density exhibit a non-analytic dependence on $1 / z$ :

$$
\beta P^{(r e g)}=\frac{1}{2} \ln (z)+\frac{1}{2 z^{1 / 2}}-\frac{1}{48 z^{3 / 2}}+\frac{3}{1280 z^{5 / 2}}+\mathcal{O}\left(\frac{1}{z^{7 / 2}}\right)
$$

and

$$
n^{(r e g)}=\frac{1}{2}-\frac{1}{4 z^{1 / 2}}+\frac{1}{32 z^{3 / 2}}-\frac{3}{512 z^{5 / 2}}+\mathcal{O}\left(\frac{1}{z^{7 / 2}}\right)
$$

which differs substantially from the asymptotical behavior in the $p<1$ case, Eqs.(51) and (52). This happens apparently because the bulk contribution to the disorder-average pressure in Eqs.(51) comes from the intervals devoid of the catalytic segments, in which reactions can not takes place and the mean density $n \sim 1$ in accordance with the Langmuir adsorption/desorption mechanism. Such intervals exist for any $p$ strictly less than unity; their contribution vanishes only when $p \equiv 1$.

\section{Conclusions.}

To conclude, in this paper we have presented an exact solution of a random catalytic reaction/adsorption model, appropriate to the situations with the catalytically-activated reactions on polymer chains containing randomly placed catalytist. More specifically, we have considered here the $A+A \rightarrow 0$ reaction on a one-dimensional regular lattice which is brought in contact with a reservoir of $A$ partilces. Some portion of the intersite intervals on the regular lattice was supposed to possess special "catalytic" properties such that they induce an immediate reaction $A+A \rightarrow 0$, as soon as two $A$ particles land onto two vacant sites at the extremities of the catalytic segment, or an $A$ particle lands onto a vacant site while the site at the other extremity of the catalytic segment is already occupied by 
another $A$ particle. For quenched random distribution of the catalytic segments, we have determined exactly the disorder-averaged pressure per site and have shown that it can be represented as a sum of a Langmuir-type contribution and a reaction-induced term. The latter can be expressed as the Lyapunov exponent of a product of random two-by-two matrices, obtained by Derrida and Hilhorst [20]. Explicit asymptotic expansions for the mean particle density and the compressibility were also derived.

\section{References}

[1] G.C.Bond, Heterogeneous Catalysis: Principles and Applications (Clarendon Press, Oxford, 1987); D.Avnir, R.Gutfraind and D.Farin, in: Fractals in Science, eds.: A.Bunde and S.Havlin (Springer, Berlin, 1994)

[2] A.Clark, The Theory of Adsorption and Catalysis (Academic Press, New York, 1970) Part II.

[3] R.M.Ziff, E.Gulari and Y.Barshad, Phys. Rev. Lett. 50, 2553 (1986); D.Considine, S.Redner and H.Takayasu, Phys. Rev. Lett. 63, 2857 (1989); E.Clement, P.LerouxHugon and L.M.Sander, Phys. Rev. Lett. 67, 1661 (1991); J.W.Evans, Langmuir 7, 2514 (1991); K.Krischer, M.Eiswirth and G.Ertl, J. Chem. Phys. 96, 9161 (1992); P. L. Krapivsky, Phys. Rev. A 45, 1067 (1992); J. W. Evans and T. R. Ray, Phys. Rev. E 47, 1018 (1993); M.Bär, N.Gottschalk, M.Eiswirth and G.Ertl, J. Chem. Phys. 100, 1202 (1994); D. S. Sholl and R. T. Skodje, Phys. Rev. E 53, 335 (1996); Yu. Suchorski, J. Beben, R. Imbihl, E. W. James, D.-J. Liu, and J. W. Evans, Phys. Rev. B 63, 165417 (2001); M. Mobilia and P.-A. Bares, Phys. Rev. E 63, 036121 (2001); P. Argyrakis, S. F. Burlatsky, E. Clément, and G. Oshanin, Phys. Rev. E 63, 021110 (2001)

[4] A.A.Ovchinnikov and Ya.B.Zeldovich, Chem. Phys. 28, 215 (1978); S.F.Burlatsky, Theor. Exp. Chem. 14, 343 (1978); D.Toussaint and F.Wilczek, J. Chem. Phys. 
78, 2642 (1983); K.Kang and S.Redner, Phys. Rev. Lett. 52, 955 (1984); J.Klafter, G.Zumofen and A.Blumen, J. Phys. Lett. (Paris) 45, L49 (1984); K.Lindenberg, B.J.West and R.Kopelman, Phys. Rev. Lett. 60, 1777 (1988); M.Bramson and J.L.Lebowitz, Phys. Rev. Lett. 61, 2397 (1988); M.Bramson and J.L.Lebowitz, J. Stat. Phys. 62, 297 (1991); S.F.Burlatsky, A.A.Ovchinnikov and G.Oshanin, Sov. Phys. JETP 68, 1153 (1989); S.F.Burlatsky and G.Oshanin, J. Stat. Phys. 65, 1095 (1991)

[5] S.F.Burlatsky and M.Moreau, Phys. Rev. E 51, 2363 (1995)

[6] G.Oshanin and A.Blumen, J. Chem. Phys. 108, 1140 (1998)

[7] S.Toxvaerd, J. Chem. Phys. 109, 8527 (1998)

[8] A.A.Naidenov and S.K.Nechaev, JETP Letters 76, 61 (2002); cond-mat/0209271

[9] G.Oshanin, S.F.Burlatsky, E.Clément, D.Graff and L.M.Sander, J. Phys. Chem. 98, 7390 (1994)

[10] J.-M. Park and M. W. Deem, Eur. Phys. J. B 10, 35 (1999)

[11] V.A.Benderskii et al, Sov. Phys. JETP 43, 268 (1976); Phys. Status Solidi B 95, 47 (1979)

[12] C.Domb, Nuovo Cimento 9, 9 (1958)

[13] H.N.V.Temperley, Proc. Phys. Soc. (London) 74, 183, 432 (1959); 77, 630 (1961); 80, $813(1962)$

[14] D.Levesque and L.Verlet, Phys. Lett. 11, 36 (1964)

[15] B.Jancovici, Physica 31, 1017 (1965)

[16] D.S.Gaunt and M.E.Fisher, J. Chem. Phys. 43, 2840 (1965)

[17] L.K.Runnels, L.L.Coombs and J.P.Salvant, J. Chem. Phys. 47, 4015 (1967) 
[18] R.J.Baxter, J. Phys. A 13 L61, (1980); R.J.Baxter, I.G.Enting and S.K.Tsang, J. Stat. Phys. 22, 465 (1980); R.J.Baxter, Exactly Solved Models in Statistical Mechanics, (Academic Press, New York, 1982); R.J.Baxter, Ann. Combin. 3, 191 (1999)

[19] J.R.Heringa, H.W.J.Blöte and E.Luijten, J. Phys. A 33, 2929 (2000)

[20] B.Derrida and H.Hilhorst, J. Phys. A 16, 2641 (1983)

[21] C.Sire and P.Krapivsky, J. Phys. A 34, 9065 (2001)

[22] A.Crisanti, G.Paladin and A.Vulpiani, Products of Random Matrices in Statistical Physics, (Springer-Verlag, Berlin, Heidelberg, 1993)

[23] R.Lima and M.Rahibe, J. Phys. A 27, 3427 (1994)

[24] B.McCoy and T.T.Wu, Phys. Rev. 176, 631 (1968); 188, 982 (1969)

[25] A.Comtet and S.Nechaev, J. Phys. A 31, 5609 (1998)

[26] H.Furstenberg, Trans. Am. Math. Soc. 108, 377 (1963)

[27] I.M.Gessel, The Electronic Journal of Combinatorics 4, R11 (1997) 